Revue européenne des sciences sociales

European Journal of Social Sciences

XXXIX-121 | 2001

L'acteur. Un concept sur la scène des sciences sociales

\title{
Les acteurs et les conséquences inattendues de leurs actions : le cas de l'université
}

Jacques Coenen-Huther

\section{(2) OpenEdition}

Journals

Édition électronique

URL : http://journals.openedition.org/ress/652

DOI : $10.4000 /$ ress. 652

ISSN : 1663-4446

Éditeur

Librairie Droz

Édition imprimée

Date de publication : 1 novembre 2001

Pagination : 125-140

ISBN : 2-600-00663-X

ISSN : 0048-8046

Référence électronique

Jacques Coenen-Huther, "Les acteurs et les conséquences inattendues de leurs actions : le cas de l'université », Revue européenne des sciences sociales [En ligne], XXXIX-121 | 2001, mis en ligne le 11 décembre 2009, consulté le 02 mai 2019. URL : http://journals.openedition.org/ress/652 ; DOI $10.4000 /$ ress.652 


\title{
LES ACTEURS ET LES CONSÉQUENCES INATTENDUES DE LEURS ACTIONS : LE CAS DE L'UNIVERSITÉ
}

\begin{abstract}
C'est probablement le sentiment de l'interdépendance des relations qu'entretiennent entre eux les êtres humains qui caractérise le mieux l'esprit sociologique ${ }^{1}$ quelles qu'en soient les manifestations. Toute sociologie favorise une vision systémique de la vie en société, même lorsque l'idée de système ne s'impose pas de manière consciente ou lorsque le mot est explicitement récusé. Qui fait profession de sociologue est généralement porté à pousser plus loin que d'autres l'examen de l'enchaînement parfois complexe des actions et de leurs conséquences, directes ou indirectes, attendues ou non, voulues ou non. Si bien que la contribution la plus spécifique de la sociologie à l'étude de toute forme d'action organisée est la mise en évidence de chaînes d'interdépendance qui ne s'imposent pas aux acteurs concernés avec toute la force de l'évidence. Bien entendu - comme le fait observer Merton dans un texte désormais classique - lorsque les conséquences des décisions et des actions correspondent effectivement aux intentions et aux anticipations des acteurs, elles peuvent être considérées comme désirables, de leur point de vue tout au moins (Merton, 1936, p. 895). C'est donc le repérage des conséquences inattendues de ces décisions et de ces actions qui peut conférer aux analyses sociologiques la plus grande pertinence sociale ou politique dans la mesure où il présente une valeur de mise en garde. Il est vrai que c'est le plus souvent a posteriori - en remontant des effets aux causes - que l'interdépendance systémique se révèle à l'observateur. C'est ce qui permet à Merton d'associer la notion de conséquence inattendue (unanticipated consequence) à celle de fonction ou de dysfonction latente $(1968$, p. 105). Mais cette identification ne peut se faire qu'après coup, en jetant un regard rétrospectif sur les événements. La capacité prédictive des sciences sociales est faible. Néanmoins, la comparaison répétée des buts que se fixent les acteurs et des résultats qu'ils atteignent ne peut qu'aider à mettre en lumière les contraintes structurelles qui pèsent sur les projets individuels ou collectifs. Elle invite à une certaine prudence dans des cas similaires et suggère certaines régularités qui, pour n'avoir pas le statut de lois, n'en offrent pas moins la possibilité de dépasser la description de l'événement singulier sur le
\end{abstract}

L'expression «esprit sociologique» est d'Eugène Dupréel, figure marquante de l'Ecole sociologique de Bruxelles, qui y voit, précisément, l'aptitude à examiner «à propos de toute question qui se pose», l'interdépendance et la complémentarité des rapports sociaux (1948, pp. 380-381). Soit dit en passant, Dupréel aimait à signaler Tocqueville comme le penseur contemporain d'Auguste Comte «le plus véritablement inspiré d'un sain esprit sociologique» (1932, Vol. 1, p. 238) sans qu'on puisse pour autant le soupçonner de participation à quelque entreprise collective d'inspiration aronienne. 
mode conditionnel, sous la forme «si A alors B ». Que de telles régularités soient envisageables n'implique pas qu'on fasse abstraction de toute autonomie individuelle et qu'on réduise l'individu à un agent totalement contraint par des forces qui le dépassent et dont il ne pourrait en aucun cas être conscient. En revanche, la formulation de propositions conditionnelles - fondées sur l'identification d'invariants du comportement humain - exige le recours à une psychologie de convention qu'on puisse attribuer à un «acteur social banalisé » ${ }^{2}$. Et l'idée même d'une mise en rapport des buts et des résultats des actions - implicite dans la notion de conséquence inattendue - requiert logiquement que l'acteur banalisé du sociologue soit un acteur intentionnel, c'est-à-dire un acteur à qui des intentions bien conscientes peuvent raisonnablement être attribuées.

Dans cette perspective, la notion de conséquence inattendue - au sens mertonien de unanticipated consequence - recouvre plus ou moins la catégorie des «effets pervers non prévus » dont parle Boudon (1979, p. 14), pour autant qu'on se limite à l'un des trois types de conséquences inattendues distingués par Merton, à savoir «the unintended consequences... which are dysfunctional for a designated system» (1968, p. 105). Il est néanmoins difficile d'accepter l'idée que ces effets non désirés puissent dans tous les cas se ramener à des effets d'agrégation ou de composition. On a certes souvent affaire à des effets « qui résultent de la juxtaposition de comportements individuels sans être inclus dans les objectifs recherchés par les acteurs» (Boudon, 1979, p. 10). Mais les effets non prévus et non désirés sont aussi très souvent des «effets de système» induits par le «surplus collectif » qu'implique l'idée même de système appliquée à un ensemble social ${ }^{3}$. Ce surplus collectif, c'est précisément ce que les sociologues appellent pour faire bref «le social» qui donne sa raison d'être à la sociologie. On l'observe déjà au niveau de la relation dyadique élémentaire. Chacun des protagonistes est porteur d'un bagage social, culturel, historique qui fait de lui ce qu'il est. Toute relation interpersonnelle est donc davantage que la rencontre de deux individualités; elle est façonnée en partie par des ressources et des contraintes préexistantes. Les composantes d'une relation sont à la fois internes et externes à cette relation ${ }^{4}$. Appliquée à une société prise dans son ensemble ou à un segment social quelconque, cette idée nous impose en bonne logique de ne pas considérer les conséquences inattendues comme la seule résultante d'effets de juxtaposition de comportements individuels mais d'y voir aussi des effets émergents du social lui-même. L'individu comme sujet n'en disparaît pas pour autant, mais il s'agit bien d'un sujet dont la liberté d'action et de réaction est soumise à des paramètres limitatifs.

D'une manière générale, les conséquences d'une action résultent de l'interaction qui s'établit entre l'action, la situation et les conditions dans lesquelles l'action est conduite (Merton, 1936, p. 895). Dès lors, plus le contexte social de l'action organisée est complexe et diversifié, plus il faut s'attendre à un degré d'opacité qui dépasse la capacité d'appréhension cognitive des décideurs intellec-

L'expression est de Raymond Boudon (1986, p. 22).

L'idée de système repose non seulement sur la notion d'interdépendance des éléments constituants, mais aussi sur l'impossibilité de réduire l'ensemble à la somme de ses parties.

4 Sur cette idée de «surplus collectif » déjà repérable au niveau de la relation dyadique élémentaire, voir: Coenen-Huther, 1998b, p. 251. 
tuellement les mieux outillés. On a d'ailleurs pu faire de cette opacité un des traits majeurs des sociétés modernes, conférant aux structures sociales un caractère impénétrable et abstrait (Zijderveld, 1974, pp. 10-12). Les conséquences d'actions même mûries avec beaucoup de soin s'enchaînent alors en de longues séquences d'interdépendance que l'esprit humain peine à anticiper dans toutes leurs ramifications. C'est la raison de l'intérêt soutenu suscité par la notion de «rationalité limitée » (bounded rationality), c'est-à-dire, en fait, de la rationalité relative à un certain cadre de référence (March et Simon, 1958, 1979, p. 136). A l'origine de cette notion, il y a, rappelons-le, la critique de la conception du décideur omniscient, apte à se donner à tout moment une vue synoptique des options possibles et l'examen de la prise de décision d'un «homme administratif», distinct de l'homo oeconomicus ou de l'acteur parfaitement rationnel (ibid., pp. 135$140)^{5}$. Bien entendu, les conséquences inattendues tendent à se multiplier lorsque les acteurs opèrent dans une configuration sociale marquée par un équilibre relatif des pouvoirs qui permet à la volonté des uns de contrecarrer à tout moment les intentions des autres. Et plus le nombre d'acteurs individuels ou collectifs est important, moins il y a de probabilité qu'un cours d'action envisagé se déroule effectivement selon le plan préconçu; ceci a constitué de longue date un argument en faveur de l'incrémentalisme dans la prise de décision (Dahrendorf, 1984, pp. 255-258), d'autant plus que le caractère inattendu de certaines conséquences produit des effets cumulatifs ne cessant de proche en proche d'éloigner l'action de son but initial. Wilhelm Wundt qui exerça une forte influence sur les sciences sociales de son temps et dont Merton note l'apport, observe à ce propos que la traduction en acte de la volonté humaine (die Betätigungen des Willens) s'effectue toujours de façon telle que les résultats des actions (die Effekte der Handlungen) dépassent dans une plus ou moins grande mesure les motivations initiales (die ursprünglichen Willensmotive); il en résulte de nouvelles motivations, sources d'actions ultérieures qui entraînent à leur tour de nouvelles conséquences (Wundt, 1886, 1903, p. 266).

L'institution universitaire actuellement en crise, au sein de laquelle un modèle organisationnel bureaucratique est en compétition incertaine avec le modèle collégial traditionnel ${ }^{6}$, est un système social en transition où les délégations d'autorité sont peu claires, les responsabilités extrêmement dispersées, voire diluées, et les capacités de blocage très fréquentes dans la mesure où nombreux sont les acteurs qui disposent de possibilités de jouer sur des facteurs d'incertitude. Il s'agit précisément d'un de ces lieux où se déroulent des séquences complexes de motivations et d'actions qui ne cessent de tromper les attentes des décideurs. On peut y observer de nombreux exemples de décisions bien intentionnées dont les conséquences inattendues sont de nature dysfonctionnelle, tant du point de vue des principes élémentaires d'une saine gestion que du point de vue de la conception classique de l'université. Il en résulte une perte de confiance dans les capacités d'autorégulation de l'institution donnant lieu à des cercles vicieux bureaucra-

\footnotetext{
5 J'ai eu l'occasion de discuter la notion de rationalité limitée dans des publications antérieures (Coenen-Huther, 1998a et 1998b).

6 Sur les conséquences de l'affaiblissement du modèle collégial traditionnel, voir: Coenen-Huther, 2000a.
} 
tiques, les tâches de coordination et de contrôle devenant à la fois de plus en plus nécessaires et de plus en plus malaisées. Examinons ici quelques processus qui affectent l'université d'aujourd'hui et qui ont entraîné les acteurs dans des voies que bien peu auraient imaginées il y a un demi-siècle.

\section{1. - LE DÉCLIN DE LA THÈSE DE DOCTORAT}

Pendant longtemps, en sciences humaines en tout cas, la thèse de doctorat fut le «chef-d'œuvre» que devait produire l'artisan intellectuel pour être admis au sein d'une communauté scientifique et être reconnu apte à faire une carrière académique au niveau le plus élevé. Le chemin qui conduisait à la soutenance publique était long et malaisé mais l'on considérait qu'il était bon qu'il en fût ainsi. Le candidat au doctorat fournissait de la sorte la preuve de sa capacité à se discipliner et à se donner progressivement les moyens de ses ambitions intellectuelles. L'entreprise révélait en quelque sorte le candidat à lui-même ${ }^{7}$. Dans le meilleur des cas, il y avait entre le directeur de thèse et le doctorant une relation de maître à disciple, mais le mûrissement de l'œuvre se faisait dans un climat d'autonomie et de responsabilité personnelle. A cela non plus, on ne voyait pas de réel inconvénient car l'élaboration de la thèse devenait ainsi une épreuve de fermeté de caractère. On ne comptait ni les heures ni les jours passés à polir son ouvrage; on ne se fixait pas non plus de calendrier trop précis: une thèse pouvait se faire en trois ou quatre ans comme en huit ou en dix. On accédait au doctorat à cinquante ans comme à trente-cinq. Ce processus de socialisation à l'ascèse intellectuelle, d'incitation au dépassement de soi-même, résista pendant un quart de siècle à la massification de l'enseignement universitaire et à l'ébranlement institutionnel issu des années soixante. Las! Au cours de la décennie écoulée, on entreprit de changer tout cela. Avec les meilleures intentions du monde, on se mit en devoir d'accélérer le mouvement, de multiplier le nombre des thésards et de fournir à ceux-ci un meilleur appui intellectuel ou de mieux les «encadrer», selon l'atroce jargon à la mode. Il s'agissait d'assurer une relève académique de qualité dans le cadre d'une institution en pleine expansion. Ainsi pourtant fut investi le dernier bastion de l'excellence académique. Les écoles doctorales qui se créent un peu partout contribuent à n'en pas douter à la scolarisation définitive de l'institution universitaire ${ }^{8}$. Les mandats d'assistants confiés à de jeunes universitaires sont attribués pour une durée strictement limitée, à la condition d'entreprendre la rédaction d'une thèse. Ils ne sont prolongés que si celle-ci progresse à un rythme soutenu. La conséquence non voulue - mais qu'on aurait pu anticiper - de cette réglementation nouvelle est de pousser bon gré mal gré vers le doctorat des jeunes gens qui n'y voient nullement l'occasion de mettre à l'épreuve une vocation mais qui désirent tout bonnement s'assurer un emploi, fût-il temporaire. Certes, on rencontre encore des doctorants par vocation - il serait très injuste à leur égard de ne

\footnotetext{
7 Dans l'esprit de «l'art de travailler» décrit par André Maurois en des termes toujours stimulants mais paraissant déjà d'un autre âge (Maurois, 1939, chap. III).

8 J'adapte ici la formulation de Richard Münch: «ihre Pädagogisierung und Umwandlung in reine Lehranstalten» (1998, p. 150)
} 
pas le souligner fortement - mais plus nombreux sont ceux pour qui la thèse n'est plus qu'une obligation professionnelle à laquelle ils se soumettent sans grand enthousiasme et qu'ils abandonnent dès qu'une autre perspective se présente. Cette situation inédite comporte une autre conséquence non voulue au départ. Une fois établi le lien entre la prolongation du contrat d'emploi et la course au doctorat, il s'impose de faire régulièrement le point sur la progression de la thèse en chantier: des procédures administratives sont prévues à cet effet. Mais le directeur de thèse est aussi l'employeur. Dès lors, l'état d'avancement des travaux du candidat est apprécié en fonction de critères qui ne sont que rarement d'ordre purement scientifique. On aura créé de la sorte une nouvelle catégorie d'universitaires au rabais: les «malgré nous » du doctorat.

\section{2. - LE CAUCHEMAR DE LA PROPÉDEUTIQUE}

Pour faire face à l'irruption en masse à l'université de jeunes gens peu préparés à y étudier avec fruit, on a imaginé diverses formules visant à combler les déficits de culture générale et à atténuer les différences entre contenu et style des études secondaires et des études universitaires. On a cru tenir la solution en transformant la première année à l'université en une propédeutique destinée à socialiser les bacheliers aux exigences de l'enseignement supérieur et à leur fournir un ensemble de connaissances de base indispensables à la poursuite de leurs études. En fait, il s'agissait de faire acquérir une culture universitaire commune à la foule de plus en plus hétérogène qui se pressait aux portes de l'université ${ }^{9}$. Il en est résulté un premier cycle aux allures monstrueuses, offrant des cours dits « de tronc commun » qui ne satisfont vraiment personne, où l'on cherche vainement le niveau de communication moyen, encore trop exigeant pour les uns, ennuyeux à force de simplification pour les autres. Des jeunes gens soucieux de bien faire et de comprendre ce qu'on attend d'eux y côtoient dans une promiscuité forcée des gamins chahuteurs à qui la taille des amphithéâtres assure l'anonymat. Il est devenu notoire que ces cours usent physiquement et psychiquement ceux qui en ont la charge sans pour autant répondre aux besoins et aux attentes de ceux qui les suivent. A vouloir supprimer le sentiment de rupture - nécessaire - entre l'enseignement secondaire et l'enseignement supérieur on en arrive à ne plus pouvoir légitimer ni défendre la spécificité de l'institution universitaire. Pour beaucoup de jeunes étudiants, cette spécificité n'est tout simplement pas comprise parce que rien n'est prévu pour la leur faire comprendre, bien au contraire. Le collège reste le monde de référence, les enseignants du niveau secondaire continuent à fournir le modèle culturel du professeur et l'université apparaît en contrepoint comme un lieu d'anomie sur lequel on se met bien vite à jeter un regard désabusé. On voit ainsi apparaître en deuxième cycle des légions d'adolescents attardés qui n'assument toujours pas leur statut d'étudiants universitaires et qu'il est déjà trop tard pour initier au «métier d'étudiant». Une fois leurs études terminées, l'université n'aura jamais été pour eux que le prolongement obligé de l'enseignement secondaire.

9 C'était le «grand dessein» - généreux - de feu Henri Janne, cofondateur avec Georges Gurvitch de l'Association Internationale des Sociologues de Langue Française (Cf. Janne, 1990, p. 172). 


\section{3. - LE MALENTENDU DES ÉVALUATIONS D'ENSEIGNEMENTS}

Lieu de création culturelle, l'université classique, on le sait, a deux fonctions appelées à se stimuler mutuellement: la recherche et l'enseignement. Dans le contexte de scolarisation de l'institution et de disparition progressive de son identité spécifique, l'opinion s'est toutefois répandue au cours des dernières décennies qu'un déséquilibre inacceptable s'était créé au détriment de la fonction d'enseignement et qu'il convenait d'y porter remède. On a fait observer que les aptitudes pédagogiques ne jouent qu'un faible rôle dans la sélection des professeurs et que les tâches d'enseignement sont relativement négligées au profit d'autres activités, intellectuellement plus gratifiantes. De bons esprits se mirent alors en devoir de stimuler de gré ou de force l'intérêt pour la fonction d'enseignement en instaurant des procédures d'évaluation des enseignants par leurs étudiants. Voilà une initiative bien raisonnable en apparence. Et pourtant, elle se révèle des plus pernicieuses dans la pratique par l'enchaînement d'effets pervers et d'intentions perverses qui est ainsi mis en branle. J'ai déjà signalé précédemment le potentiel de chantage moral et de laxisme éducatif que recèle cette innovation pédagogique, en principe judicieuse, alors que l'université est confrontée à des générations élevées en très grande majorité dans un climat de permissivité confinant souvent à la démission éducative ${ }^{10}$. Face à des jeunes gens qui n'ont guère pris l'habitude de se voir opposer une volonté ferme ni imposer des règles strictes, on décourage ainsi toute velléité que pourrait avoir le professeur de jouer un rôle d'agent socialisateur. L'épée de Damoclès de l'évaluation défavorable menace en effet chaque membre du corps enseignant, d'autant plus que les données d'évaluation recueillies ne sont pas livrées exclusivement aux réflexions de l'intéressé mais sont utilisées pour comparer entre eux les enseignants d'un même département. Dès lors, toute tentative quelque peu vigoureuse de restaurer la conception classique de l'université comme foyer de culture ne peut que susciter des réactions de mauvaise volonté de la part de jeunes peu doués ou peu motivés et se traduire par la baisse du score d'appréciation du professeur téméraire qui s'y risque; on ne manque pas de lui attribuer des difficultés de contact avec son auditoire. Dans un milieu professionnel caractérisé par l'individualisme et la compétition, il n'est aucune solidarité ni compréhension à attendre des collègues; aucun secours non plus. Les «problèmes avec les étudiants» - comme tout autre problème qu'on n'arrive pas à dissimuler sous un masque d'assurance tranquille - ne suscitent qu'un sentiment de Schadenfreude trop évident. Ils peuvent en outre être utilisés de manière ad hoc dans des jeux de pouvoir qui seraient futiles - tant les enjeux sont dérisoires - s'ils ne mettaient pas cyniquement en jeu l'avenir professionnel et scientifique des uns et des autres. Dès lors, les manifestations les plus éhontées d'irresponsabilité, de paresse intellectuelle, d'absence de motivation pour les études, de manque de respect pour l'institution et ceux qui la représentent sont acceptées avec une placidité qui se donne l'allure de l'indifférence. En bref, on voulait promouvoir davantage de considération pour les tâches d'enseignement; on a contribué à accentuer la délégitimation de la fonction d'enseignant qui

10 Voir à ce sujet: Jacques Coenen-Huther, 2000b, p. 96. 
affecte de nos jours tous les niveaux du système d'enseignement depuis l'école primaire jusqu'à l'université. On est bien loin du «climat de partenariat entre enseignants et enseignés» invoqué démagogiquement par le rapport Kleiber (p. 120).

\section{4. - LES ILLUSIONS DE L'ÉGALITARISME}

Traditionnellement, les diverses formes de contrôle des connaissances instaurées par l'institution universitaire ont fait l'objet d'une note. Sous sa forme chiffrée, cette note a constitué pour des générations d'étudiants et d'enseignants le condensé d'une appréciation: excellente, médiocre, insuffisante, etc. Il arrivait que le niveau de la note fût contesté mais le système en soi n'était pas remis en question: de part et d'autre, on le jugeait commode. Une note s'approchant dangereusement de la limite acceptable avait valeur de clignotant; une note inférieure à la moyenne indiquait une lacune à laquelle il devenait urgent de porter remède. Il y a environ deux décennies, à l'université comme aux autres niveaux du système d'enseignement, des réformateurs bien intentionnés entamèrent une campagne contre le principe même de la note. Les motivations invoquées étaient diverses: le système était faussement objectif, l'appréciation ainsi formulée était trop sommaire, les notes les plus basses avaient quelque chose d'inutilement brutal, enfin - et surtout - le recours au chiffre recouvrait un classement individuel condamné par un égalitarisme naïf. Peu à peu, l'abandon de la note devint le cheval de bataille des enseignants qui se voulaient modernes, novateurs, tournés vers l'avenir. Le remplacement du système qui avait fait ses preuves par une formule d'appréciation non chiffrée du type «acquis/non acquis » fut considéré comme une réforme appelée à se généraliser, tant elle semblait conforme à l'esprit du temps. Pourtant, là où elle fut appliquée, cette innovation révéla rapidement ses inconvénients. L'appréciation non chiffrée est frustrante pour les meilleurs étudiants - ceux qui restent fidèles à l'idéal d'excellence de l'institution universitaire - car leurs performances ne sont pas mises en valeur; elle favorise en revanche les étudiants médiocres dont elle gomme les insuffisances. Au surplus, en réduisant les résultats de l'appréciation à une dichotomie peu explicite, ce système exige des commentaires circonstanciés dont la note constituait précisément le résumé. Dans une conjoncture marquée par le gonflement des effectifs estudiantins, il devient de plus en plus difficile d'offrir de tels commentaires à chaque étudiant. Dès lors, ils ne sont plus fournis qu'aux étudiants particulièrement tenaces ou particulièrement revendicatifs. Les autres - la grande masse - ont le sentiment d'être confrontés à une très grande indifférence de la part du corps enseignant. Un cercle vicieux en résulte: les efforts pour briller se font de plus en plus rares parmi les étudiants; le manque d'intérêt va croissant au sein du corps enseignant.

\section{5. - LES MIRAGES DU TOURISME ESTUDIANTIN}

Au cours de la décennie écoulée, un peu partout en Europe, on se mit en devoir de favoriser ce qu'on convient d'appeler «la mobilité estudiantine». Il s'agissait d'encourager les étudiants universitaires à se rendre dans une université étrangère pour y suivre des cours pendant un ou deux semestres et de leur faciliter de tels 
déplacements. L'idée était incontestablement séduisante; elle renouait avec la tradition des voyages en Italie des bacheliers d'autrefois. Pour la rendre praticable et pour éviter que les candidats à l'aventure européenne ne se retrouvent pénalisés par leur initiative, une certaine harmonisation des procédures et des programmes s'imposait toutefois. Ce fut un puissant incitant à l'introduction des crédits d'études transférables dans le cadre de l'ECTS (European Credit Transfer System) et à la semestrialisation des enseignements. Quoi de plus neutre et de plus innocent en apparence que de tels aménagements d'ordre purement pratique! En fait, sans que personne ne l'ait voulu - dans un contexte qui n'est plus celui des vagabondages intellectuels d'Erasme - toute l'entreprise contribua à l'accélération de la dégradation de l'enseignement supérieur ${ }^{11}$. La semestrialisation - on s'en rend compte à présent - n'est rien d'autre que le coup de grâce porté à l'esprit de l'enseignement universitaire. Quand un cours se termine à quelques jours du début d'une session d'examens comme c'est la règle dans un programme semestrialisé, on ne peut guère s'attendre à ce que les étudiants fassent un effort d'approfondissement personnel et se livrent à quelque lecture complémentaire audelà du minimum imposé, à supposer qu'ils en aient envie. La chasse aux crédits d'études fait passer au second plan le souci de la note: que celle-ci reflète l'excellence ou la médiocrité, peu importe pourvu qu'on ait les crédits! Quant aux séjours à l'étranger, ils ne sont que trop souvent l'occasion de ramener dans son université d'origine des crédits d'études obtenus dans des conditions douteuses; tout pousse en effet à accorder à l'étudiant en déplacement le bénéfice du doute lorsque ses performances sont peu convaincantes. Enfin, contrairement aux formations postgrades dans des institutions de pointe, les déplacements estudiantins en cours d'études compliquent l'élaboration de programmes rationnellement conçus. De nombreux enseignants universitaires en conviennent, mais les gestionnaires malavisés qui se sont donnés pour tâche de réformer l'université tendent à faire de la proportion d'étudiants en déplacement un indice de dynamisme lors des évaluations de départements universitaires. Sur ce point, le rapport Kleiber nous livre pourtant un aveu de taille dont son auteur ne perçoit sans doute pas bien la portée. On y lit en effet ceci: «Quand on leur demande enfin quelles ont été les motivations qui les ont poussés à bouger, les étudiants mettent presque systématiquement en avant des motifs d'ordre personnel ou culturel ${ }^{12}$, bien avant ceux liés à la formation » (p. 108; souligné par JCH). Ils reviennent en effet dans leur université d'origine avec «l'expérience d'un vécu différent» (ibid.) mais guère plus avancés dans leurs études, parfois de leur propre aveu.

11 Et que telle ou telle faculté se targue de succès en la matière ne change rien au diagnostic global qui peut être porté sur l'entreprise (Cf. Davaris, 2001).

12 «Ah! qu'en termes galants ces choses-là sont mises!», comme aurait dit Molière. En pratique, cela signifie par exemple qu'on choisit une université de niveau médiocre dans un ville réputée attirante plutôt que des enseignements de qualité offerts sur un campus isolé de l'agitation urbaine. Cela peut signifier aussi qu'on se décide en fonction de la langue et du climat: l'Espagne, plutôt que la France, l'Angleterre ou l'Allemagne, pour y parfaire des études en sciences sociales; mais en Andalousie plutôt qu'à l'Universidad Complutense. 


\section{6. - LE CHAOS DES PROGRAMMES «À LA CARTE»}

Dans le souci de rendre l'enseignement universitaire plus attrayant et d'augmenter les possibilités de choix des étudiants, on a rendu, dans de nombreuses disciplines, le cursus beaucoup plus souple qu'il ne l'était autrefois. La succession rigide des programmes annuels a fait place, après l'année propédeutique, à un «deuxième cycle» offrant de multiples possibilités de parcours individuels où l'ordre des cours à prendre est largement laissé à l'appréciation des étudiants euxmêmes. Dans le même esprit, on a réduit le nombre de cours obligatoires et on a multiplié le nombre de cours à option. Dans ce contexte - bel exemple de «frustration relative»-l'idée même de prérequis imposé pour s'inscrire à certains cours tend de plus en plus à être ressentie comme une contrainte insupportable par les jeunes gens qui ont à se constituer leur programme «à la carte». Après une décennie d'application de ce nouveau mode d'organisation des plans d'études, ses conséquences non voulues et non anticipées sont devenues évidentes. Le fardeau administratif est énorme, la difficulté de dispenser des enseignements adaptés au niveau de connaissance d'auditoires de plus en plus hétérogènes va croissant, les choix des étudiants sont dominés par la course aux crédits d'études plus souvent que par le souci d'accumuler des connaissances. Tout cela, combiné avec la semestrialisation des cours, donne des résultats proprement désastreux. Les trois premières semaines d'un semestre universitaire qui en comporte quatorze sont marqués par les allées et venues d'étudiants indécis qui n'ont pas encore composé définitivement leur programme personnel. On «vient voir»... si le cours n'est pas trop difficile, si la matière n'est pas trop aride, si l'enseignant n'est pas trop exigeant... on repart, on revient. Le renouvellement hebdomadaire des auditoires peut affecter jusqu'à un tiers des effectifs avant que la situation ne se stabilise. Les deux dernières semaines du semestre sont marquées par une nervosité croissante à l'approche imminente des examens. Il reste en tout et pour tout neuf semaines qui peuvent être consacrées à l'enseignement dans une atmosphère plus ou moins sereine.

Interrogeons-nous à présent sur les raisons de cette avalanche peu banale de conséquences inattendues, dans un contexte organisationnel où les compétences décisionnelles ne manquent pourtant pas. Dans son analyse classique déjà citée, Merton note que l'obstacle le plus évident à une anticipation correcte des conséquences d'une action est à rechercher dans l'état de nos connaissances (the existing state of knowledge). Il admet néanmoins que le défaut de connaissances adéquates ne constitue la seule barrière que dans les cas les plus simples (Merton, 1936, p. 898). Les exemples brièvement décrits plus haut ne relèvent pas des cas de figure les plus simples car ils mettent en jeu des systèmes d'actions coordonnées. Examinons-les toutefois à la lumière des catégories de facteurs dont Merton estime qu'ils contribuent à une connaissance inadéquate (ibid). Nous allons voir qu'il est difficile de s'en tenir strictement au plan cognitif. Merton ne s'y tient d'ailleurs pas luimême puisqu'il fait intervenir des «basic values» dans son analyse (ibid., p. 903).

Le déclin de la thèse de doctorat reflète le plus directement le déclin de la conception classique de l'université que j'ai déjà évoqué ailleurs ${ }^{13}$. La prise de

13 Voir à ce sujet: Coenen-Huther, 2000b. 
décision en ce domaine est fortement influencée par les instigations des instances de financement qui se muent progressivement en instances de contrôle ${ }^{14}$. Cellesci, toujours en quête d'indicateurs de performance prétendument objectifs, incluent le nombre de thèses et le rythme de leur achèvement dans leurs critères d'appréciation. Comme en d'autres matières, on sent ici la fascination exercée par le modèle des sciences de la nature et par l'exemple de disciplines où le doctorat n'a pas du tout la signification ni l'importance qu'il a eu en sciences humaines. Il y a une quinzaine d'années, un recteur de l'Université de Genève, physicien de formation, illustra cette conception expéditive de manière particulièrement abrupte par le slogan «la matu à 17 ans; la thèse à 25 ans ${ }^{15}$. On en fit des gorges chaudes à l'époque, mais force est bien de constater que l'évolution en cours se rapproche dangereusement de ce scénario, peut-être envisageable dans un département de physique mais ne pouvant qu'être tenu pour caricatural dans les facultés de sciences humaines. A cet égard, la soumission empressée de certains responsables facultaires aux injonctions bureaucratiques extérieures donne à penser que la scolarisation de l'institution universitaire n'est plus pour eux un objet de débats. Ils en ont d'ores et déjà admis le principe. Merton verrait dans cette attitude le résultat d'une préoccupation majeure pour les avantages les plus immédiats et les plus tangibles - en l'occurrence, la bonne tenue des statistiques universitaires - au détriment des conséquences à plus long terme (Merton, 1936, pp. 901-902).

Les dysfonctions au stade du premier cycle marquent l'échec de l'idée de propédeutique dans les conditions actuelles. Ces dysfonctions sont bien évidemment les conséquences directes de la massification de l'enseignement supérieur et de l'accès généralisé aux facultés universitaires imposé par l'autorité politique. L'idée d'assurer un complément de formation générale à un public hétérogène au sein duquel les prérequis indispensables font le plus souvent défaut était certes généreuse mais sans doute peu réaliste. Ici, il y a clairement interférence de ce que Merton qualifie de nécessité induite par certaines valeurs (1936, p. 903). Quant aux efforts ${ }^{16}$ entrepris pour atténuer le sentiment de rupture entre l'enseignement secondaire et l'université, ils ne peuvent qu'être neutralisés par les formules de «tronc commun » qui, au contraire, projettent brutalement les jeunes collégiens dans un monde totalement différent de ce qu'ils ont connu précédemment. Il apparaît de plus en plus clairement qu'on s'est donné ici des objectifs parfaitement contradictoires. La confusion mentale résulte en l'occurrence de l'acceptation résignée d'une massification envahissante et désordonnée, tenue pour inéluctable. Observons au passage que rien ne justifie un tel fatalisme. La situation actuelle est clairement le résultat non prévu et non voulu d'une visée politique dépassant largement le cadre de l'enseignement supérieur. Pourquoi la constatation de ses effets dévastateurs ne déboucherait-elle pas sur une volonté politique nouvelle,

14 Au mépris de plus en plus ouvertement affiché de la liberté académique.

15 La «matu », c'est-à-dire « la maturité », étant l'équivalent du baccalauréat dans le système scolaire genevois.

16 Efforts encore en cours à l'Université de Genève sous forme d'expériences-pilotes aux dimensions dérisoires. 
mieux avisée que par le passé17? La diversification de l'enseignement supérieur qui est en ce moment à l'ordre du jour en offre l'occasion.

La généralisation irréfléchie de l'arme dangereuse des évaluations d'enseignements repose de toute évidence sur l'oubli du contexte éducatif général favorisant la «culture du narcissisme» (Lasch, 1979) et de l'état des relations entre générations qui en résulte. Cette mesure se fonde plus particulièrement sur une image fausse des étudiants d'aujourd'hui - leurs besoins, leurs aspirations, leurs représentations de l'enseignement universitaire, leur relation à l'autorité -, sur une conception erronée de la fonction enseignante assimilée de façon simpliste à l'offre d'un service à une clientèle (Coenen-Huther, 2000b, pp. 93-95) et sur une vision irénique de l'institution universitaire. Celle-ci est en effet en proie à des rivalités internes qui n'ont rien à voir avec la promotion des qualités pédagogiques et qui font du questionnaire d'évaluation un instrument redoutable ${ }^{18}$. On se trouve ici devant un véritable cas d'école de prise de décision fondée sur une conception systémique inadéquate. L'environnement du système social en question est totalement négligé; les tensions internes à ce système le sont tout autant. Dans les termes de Merton, on pourrait certainement parler à ce propos de «connaissance partielle» (partial knowledge) conduisant à une appréciation erronée de la situation (1936, pp. 899-901).

Les velléités d'abandon du système de notation chiffrée résultent de l'égalitarisme irréaliste qui, de façon plus générale, sape la légitimité de l'institution universitaire comme centre d'excellence. Que des enseignants universitaires s'en fassent les champions indique bien à quel point la conception classique de l'université est déjà minée de l'intérieur. Ceci n'a rien de trop surprenant si l'on songe que la massification de l'enseignement supérieur n'a pas affecté uniquement la population estudiantine mais aussi le corps professoral. Pour parler clair, la médiocrité a envahi l'institution par les deux extrêmes de la hiérarchie universitaire. Quoi qu'il en soit, c'est l'idée même d'aspiration à l'excellence qui s'en trouve disqualifiée. Sur ce point, ce sont deux principes sous-jacents aux conceptions égalitaristes qui s'opposent en fait l'un à l'autre. Le souci d'éliminer l'esprit de compétition est basé sur la notion implicite d'une qualité du social à préserver ou à restaurer; celle-ci se trouverait menacée par la mise en évidence d'inégalités de performance qu'il serait préférable de camoufler pudiquement. Mais on en arrive ainsi à négliger l'idée de mérite qui est cependant aussi aux fondements de l'égalitarisme moderne. Cette contradiction est bien dans l'air du temps et procède d'idées reçues comportant une part d'ambiguïté. De nos jours, en effet, l'égalité est une valeur dominante, au point qu'elle est présumée juste et que c'est l'inégalité qui doit être justifiée. Mais cette valeur est dans un état de tension permanente avec les aspirations individualistes qui exigent que le mérite individuel soit

17 Une commission d'experts incluant l'auteur de ces lignes, agissant à la demande du Vlaamse Interuniversitaire Raad [Conseil Interuniversitaire Flamand], a recommandé récemment l'instauration d'une «épreuve d'orientation obligatoire à forte valeur indicative » pour canaliser l'accès aux enseignements de sociologie et de science politique dans les universités flamandes (VLIR, 2000, p. 17). La presse flamande s'est faite largement l'écho de cette recommandation.

18 Le rapport déjà cité recommande que les évaluations d'enseignements soient menées dans le respect « de la liberté créative et de la dignité des enseignants» (VLIR, 2000, p. 19). 
reconnu et valorisé ${ }^{19}$. Les «basic values» évoquées par Merton sont certainement en cause, mais il y a lieu de souligner également à ce propos que nos actions n'ont pas toujours de but clairement définissable, celui-ci étant «more often than not nebulous and hazy », ce qui est bien le cas ici (1936, p. 896).

L'engouement récent pour la «mobilité estudiantine» est principalement le fait d'une cécité à l'égard des effets de système qu'entraîne cette innovation. Les brefs séjours d'études d'un ou deux semestres à l'étranger rendaient une certaine harmonisation des procédures indispensable. Il en est résulté l'introduction des crédits d'études transférables. Ceux-ci impliquaient à terme la semestrialisation qui, à son tour, pèse sur les modes de contrôle des connaissances. Les examens succédant immédiatement aux derniers cours, à la fin de chaque semestre, ne posent peut-être pas de problèmes lorsqu'il s'agit d'enseignements à caractère technique pour lesquels l'assiduité au cours est le principal gage de succès et qui pourraient tout aussi bien trouver leur place dans une Haute Ecole Spécialisée. Ils ne peuvent qu'entraîner une baisse du niveau des exigences pour les matières qui relèvent d'un cursus proprement académique. Une fois de plus, c'est l'essence même de l'enseignement universitaire qui est méconnue. En référence à l'analyse mertonienne, le défaut d'anticipation n'est pas dû ici à une connaissance insuffisante mais plus particulièrement à un type de connaissance (type of knowledge) qui tend à sous-estimer l'importance des enchaînements de facteurs (Merton, 1936, p. 898).

La promotion des programmes d'études «à la carte» procède de la même incapacité à anticiper les interdépendances systémiques affectées par une innovation qui est à la fois pédagogique et organisationnelle. Elle est fondée sur une sousestimation radicale des difficultés de sa mise en œuvre. Un peu partout, on s'interroge d'ailleurs sans trop oser le proclamer ouvertement sur les moyens de réduire la portée de cette réforme qui est en train de se muer en cauchemar administratif. Mais l'idée de la multiplication des options - introduite en Europe alors qu'on commençait, aux Etats-Unis, à en percevoir les inconvénients en termes de lacunes et de déséquilibres dans la formation - repose avant tout sur l'attribution aux étudiants d'une rationalité qui n'est en aucune façon la leur. Dans leur grande majorité, les étudiants, répétons-le, ne sont pas ces jeunes gens avisés, évoqués par le rapport Kleiber (p. 34). Certes, l'enseignement universitaire a été organisé traditionnellement autour de la présupposition que les étudiants sont de jeunes adultes raisonnables ayant le souci à moyen terme de la meilleure formation possible. Et il importe de souligner que cette présupposition reste absolument indispensable. Mais il faut bien voir qu'elle est largement de l'ordre de la fiction. Cette fiction doit être maintenue dans les rapports entre le corps professoral et la population estudiantine en raison de sa valeur éducative irremplaçable. Les apôtres de la plus grande liberté de choix sont néanmoins tombés dans le piège qui consiste à confondre le normatif et le réel. On ne peut espérer trouver chez la plupart de ces jeunes gens une rationalité d'adulte d'âge mûr et attendre d'eux des stratégies correspondantes. Mais la confusion de la norme et de la réalité introduit nolens volens

19 D’où le reproche fait par Julien Benda aux «égalitaristes modernes » d'avoir cessé de comprendre «qu'il ne peut y avoir d'égalité que dans l'abstrait et que l'essence du concret est l'inégalité» (1927, p. 100) 
un fait nouveau dans la situation réelle (Merton, 1936, pp. 903-904). En laissant le champ libre à ce qu'on doit bien souvent tenir pour des caprices d'enfants gâtés, elle écarte davantage encore l'institution universitaire de sa vocation initiale.

Les réflexions qui précèdent ne peuvent que nous amener à reconsidérer plus attentivement la notion de rationalité limitée. Celle-ci, comme on l'a rappelé plus haut, est née de la mise en évidence du caractère fallacieux de la vision synoptique qui fournirait la base de la prise de décision. Le décideur n'est pas cet individu qu'on l'appelle acteur, agent ou sujet - qui «a étalé devant lui l'ensemble complet des possibilités de choix parmi lesquels il élira son acte» (March et Simon, 1958, 1979, p. 135). Comme je l'ai suggéré ailleurs, la rationalité limitée n'est pas une forme spécifique de rationalité mais bien l'introduction d'une clause restrictive pouvant affecter tout modèle de comportement mettant en scène un acteur intentionnel (Coenen-Huther, 1998b, p. 257). La présentation la plus courante du modèle décisionnel orienté par une forme de rationalité limitée met l'accent sur les aspects cognitifs de la prise de décision. On se trouvera ainsi en situation de certitude, en situation de risque ou en situation d'incertitude (March et Simon, Ibid.). Par ailleurs, les options possibles ne se présentent pas à l'acteur de façon synchronique mais de façon séquentielle, ce qui tend à relativiser très nettement l'idée d'optimisation. La recherche de la solution optimale se rapporte toujours à un optimum dans les conditions du moment (ibid., p. 136; Coenen-Huther, 1998b, p. 258). Mais les paramètres limitatifs qui affectent les choix des acteurs et les placent en état de lucidité partielle ${ }^{20}$ ne sont pas exclusivement d'ordre cognitif. Certes, les théoriciens de la rationalité limitée introduisent dans les déterminants du choix un schéma simplifié qu'ils qualifient de «définition de la situation». Ils admettent également que les éléments de la définition de la situation ne sont pas des «données» mais sont «eux-mêmes le produit de processus psychologiques et sociologiques comprenant les activités propres de celui qui choisit et celles des autres dans son milieu» (March et Simon, 1958, 1979, p. 137). Mais il importe d'être plus précis quant aux ingrédients de la définition de la situation. La manière dont les acteurs définissent une situation et y réagissent n'est pas seulement le résultat d'une combinaison de connaissances, de motivations et de représentations. Il faut y inclure des jugements de valeur et des options idéologiques plus ou moins cristallisées qui orientent la réception sélective des informations.

Les processus envisagés plus haut impliquent certes des options fondées sur une information inadéquate: ainsi en est-il par exemple du type de rationalité attribué à la population estudiantine ou de la généralisation abusive du modèle des sciences de la nature. Mais l'information n'est pas seule en cause. Des représentations sédimentées de longue date - représentations des étudiants, de la science, des disciplines universitaires - donnent à penser aux acteurs concernés qu'ils sont suffisamment et adéquatement informés. Dans d'autres cas, c'est le mode de conceptualisation de l'objet de la prise de décision qui est en cause: l'inaptitude à concevoir les interdépendances systémiques, l'absence de prise en compte du contexte du système ou de ses tensions internes, les analogies fausses. On reste ici dans le domaine cognitif, mais il n'est pas davantage question de réduire tout ceci

20 Par rapport à un Deus ex machina dont l'intervention aurait assuré l'élimination de toute conséquence inattendue. 
à l'information des décideurs; il s'agit bien d'une certaine manière de raisonner, fruit d'une formation préalable, difficile à communiquer à ceux qui ne bénéficient pas d'une formation semblable.

Représentations et procédés de raisonnement acquis antérieurement jouent donc un rôle dans l'attitude adoptée à l'égard des éléments d'informations dont on dispose. Mais - on l'a vu à plusieurs reprises - les orientations adoptées se sont fondées sur une certaine conception, implicite ou explicite, de l'institution universitaire et de sa mission à notre époque. Quant aux objectifs contradictoires, aux entreprises impossibles, aux refus d'envisager certaines solutions, ils sont clairement influencés par des éléments idéologiques, même si ceux-ci sont loin d'être toujours explicitement formulés. Crozier et Friedberg ont raisonné à cet égard en termes de contraintes - culturelles et structurelles - pesant sur les choix des acteurs (1977, pp. 276-281). Peut-être est-il possible de généraliser davantage en évoquant non plus les contraintes mais les composantes cognitives, structurelles et socioculturelles des processus de prise de décision.

Département de Sociologie

Université de Genève

\section{RÉFÉRENCES BIBLIOGRAPHIQUES}

Benda, Julien, La trahison des clercs, Paris, Bernard Grasset, 1927.

Boudon, Raymond, Effets pervers et ordre social, Paris, Presses Universitaires de France, 1979.

Boudon, Raymond, L'idéologie. L'origine des idées reçues, Paris, Fayard, 1986.

Coenen-Huther, Jacques, La sociologie face au changement social, in «Revue Européenne des Sciences Sociales », Tome XXXVI, No 110, 1998a, pp. 121-134.

Coenen-Huther, Jacques, Relation interpersonnelle, interaction et transaction, in « Revue Européenne des Sciences Sociales », Tome XXXVI, No 112, 1998b, pp. 247-262.

Coenen-Huther, Jacques, Stratégies de dissimulation et rationalité limitée, in: André Petitat, Ed., «Secret et lien social», Paris, L'Harmattan, 2000a, pp. 137-146.

Coenen-Huther, Jacques, Les sociologues et la crise de l'université: peur, aveuglement ou complicité?, in «Revue Européenne des Sciences Sociales», Tome XXXVIII, No 119, 2000b, pp. 89102.

Crozier, Michel et Friedberg, Erhard, L'acteur et le système. Les contraintes de l'action collective, Paris, Seuil, 1977.

Dahrendorf, Ralf, Reisen nach innen und außen. Aspekte der Zeit, Stuttgart, Deutsche Verlags-Anstalt, 1984.

Davaris, Sophie, La Faculté de Droit à la pointe des échanges Erasmus, in «Campus. Magazine de l'Université de Genève », No 50, février-mars 2001, pp. 26-27.

Dupréel, Eugène, Traité de morale, 2 vol., Bruxelles, Editions de la Revue de 1'Université de Bruxelles, 1932.

Dupréel, Eugène, Sociologie générale, Paris, Presses Universitaires de France, 1948.

Janne, Henri, Maîtriser la mouvance des structures de l'Université, in «L'Université en question», AISLF, Toulouse-Le Mirail, 1990, pp. 169-173.

Kleiber, Charles, Pour l'Université, s.l.n.d.

Lasch, Christopher, The Culture of Narcissism. American Life in an Age of Diminishing Expectations, New York, Norton and Company, 1979. 
March, J.G. et Simon, H.A., Organizations, New York, John Wiley and Sons, 1958. Tr. fr. Les organisations. Problèmes psycho-sociologiques, Paris, Dunod/Bordas, 1979.

Maurois, André, Un art de vivre, Paris, Plon, Coll. «Présences», 1939.

Merton, Robert K., The Unanticipated Consequences of Purposive Social Action, in «American Sociological Review», Vol. 1, 1936, pp. 894-904.

Merton, Robert K., Social Theory and Social Structure, Enlarged edition, New York, The Free Press, 1968.

Münch, Richard, Editorial, in «Soziologische Revue», Vol. 21, No 2, 1998.

VLIR (Vlaamse Interuniversitaire Raad), De onderwijsvisitatie Politieke en Sociale WetenschappenSociologie. Een onderzoek naar de kwaliteit van de opleidingen Politieke en Sociale Wetenschappen en Sociologie aan de Vlaamse universiteiten [La visite des enseignements de sociologie et de sciences sociales et politiques. Une enquête sur la qualité des formations en sociologie et en sciences sociales et politiques dans les universités flamandes], Bruxelles, décembre 2000.

Wundt, Wilhelm, Ethik, Stuttgart, Enke Verlag, 1886. Deuxième édition, Vol. 1, 1903.

Zijderveld, Anton C., The Abstract Society, Harmondsworth, Penguin Books, 1974. 